\title{
The compressive mechanical properties and constitutive relation of polyurethane elastomer
}

\author{
Shengjia Chen ${ }^{1 \mathrm{a}}$, Zhigang $\mathrm{Li}^{2 \mathrm{~b}}$ \\ ${ }^{1}$ Taiyuan University of Technology, Jinzhong 030600, China; \\ ${ }^{2}$ Taiyuan University of Technology, Jinzhong 030600, China. \\ achenshengjia_tyut@163.com, ${ }^{\text {b }}$ Ixlizhigang@163.com
}

Keywords: Polyurethane elastomer, quasi-static compression experiment, the constitutive model.

\begin{abstract}
In order to describe the mechanical property of polyurethane elastomer (PU elastomer) under quasi-static compression, we have adopted universal tester of South JingKe electronics to proceed with equivalent static compression for polyurethane elastomer material, and studied the mechanical behavior of PU elastomer under the rate of $1.2 \mathrm{~mm} / \mathrm{min}$, namely the corresponding strain rate $10^{-2} / \mathrm{s}$. The results show that: when the strain is between 0 and 0.2 , the slope of curve decreases with increasing strain, and the variable quantity is extremely small; between 0.2 and 0.6 , it continues to increase with increasing strain, presenting the feature of up concave and showing hardening phenomenon; and it maintains rubber elasticity in the entire deformation process. In order to get reasonable constitutive model of PU elastomer, we also need to meet the conditions of large compressive deformation, the Neo-Hookea model, Mooney-Rivilin model, Yeoh strain-energy function, improving Mooney-Rivilin model and James-Green-Simpson form was choose which matching of experimental data, finally obtain the constitutive model of PU elastomer material under quasi-static compression, and determine the stress-strain relation.
\end{abstract}

\section{Introduction}

PU elastomer with good mechanical properties, such as high strength, low thermal conductivity, as well as outstanding overall performance, makes itself can be applied in a quite broad range, which can be used for potting and cladding products, sealing, rubber tires, conveyor belts and so on [1]. However, whether it is applied in high pressure seal, shock absorbers or vehicle bumper material, or it is used as the sealing material for the machine, and PU elastomer material applied in all kinds of product applications is compressed with inevitable destruction, so the study on the compressive mechanical properties of rubber has great engineering significance.

Currently the constitutive model of rubber material can be divided into phenomenal model based on strain-energy function and statistical model based on molecular chain network [2-5]. In order to make better use and design of the rubber components, it is necessary to have in-depth understanding of the mechanical properties of rubber, so the study of the mechanical properties of polymer materials is done by some scholars. In 2003, Song et al. [6] based on the quasi-static and dynamic experiments achievements, established new model of stress-strain behavior related to homotaxial strain-rate when the EPDM rubber is in compression or tension. In 2009, Zhao Hua et al. [7] based on the rate related properties of the polyurethane elastomer material at the compression deformation, established a nonlinear viscoelastic constitutive model of polyurethane elastomer material and used numerical analysis to verify the validity of the model. In 2013, Wu Kaisong et al. [8] proposed the idea of using the rubber deformation incompressible way to handle test data so as to improve the accuracy of the constitutive relation, and respectively used traditional methods and large deformation incompressible way to process test data of nitrile rubber in quasi-static compression, and then based on Yeoh strain energy function, validated that large deformation incompressible method can effectively reduce the errors existing in the fitting constitutive model. However, few studies are done on the compression mechanical properties of PU elastomers in applications, so the compressive mechanical behavior and constitutive model of the PU elastomers are the main research subjects in this dissertation. 


\section{The experimental process}

The experiment adopts universal tester of South JingKe electronics (20KN sensor) to proceed with quasi-static compression experiment for polyurethane elastomer material, and obtains the material's stress-strain relation at the strain rate $10^{-2} / \mathrm{s}$. Conduct an experiment in accordance with GB/T7757-2009 [9], and experimental material is polyurethane elastomer, the density and hardness of the rubber is $1.2 \mathrm{~g} / \mathrm{cm}^{3}$ and (Shore A): $60 \pm 5$ respectively, the specimen size is designed as diameter $10 \mathrm{~mm}$ and thickness $2 \mathrm{~mm}$, the experimental process is that use rubber sample to do 5 times experiments at corresponding strain rate, and finally take the average value of the 5 experimental results as the final value as well as obtain the raw data of compression test. After processing, get stress-strain curve of PU elastomer under quasi-static compression.

\section{The experimental results and the analysis}

According to GB/T7757-2009 [9] experimental data processing method, in the compression test, the test-piece's compressive stress is $\sigma_{e}$ and strain is $\varepsilon_{e}$, we can obtain by the formula:

$$
\sigma_{e}=\frac{F}{A}, \quad \varepsilon_{e}=\frac{\Delta h}{h_{0}}
$$

$F$ is the axial force applied to the specimen, $A$ is the test-piece's initial cross sectional area, $\Delta h$ is the test-piece's axial compress variation, $h_{0}$ is the test-piece's initial axial length. Figure 1 (a) shows the rubber's compressive load displacement curve, then it is able to get the experiment's stress-strain curve shown in Figure 1 (b).

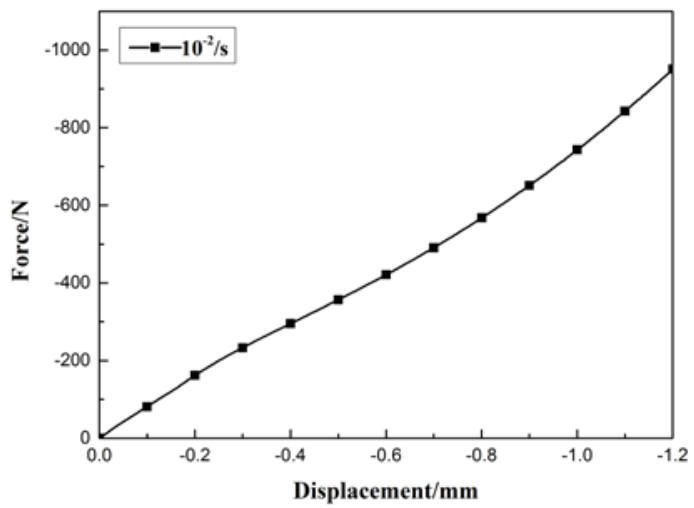

(a) The load-displacement curve

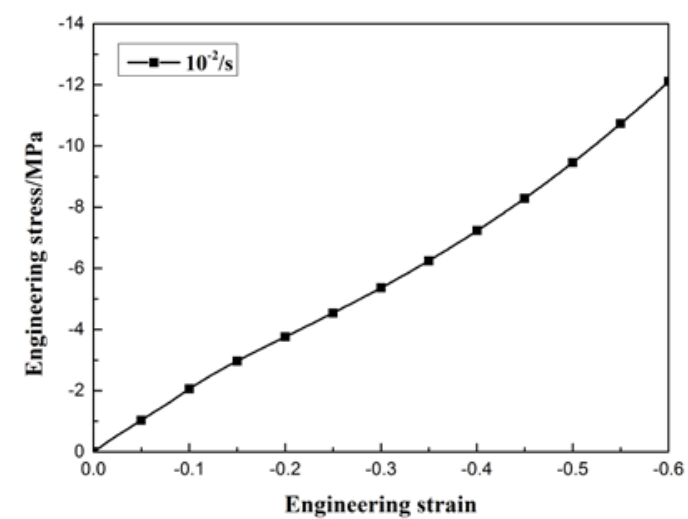

(b) The stress-strain curve

Fig. 1 The compression experiment results of PU elastomer

During the experiment process, short cylinder test-piece presents drum type, it can be seen the stress-strain curve is nonlinear, thus the material of PU elastomer is nonlinear; when the strain is from 0 to 0.2 , the slope of curve decreases with increasing strain, and the variable quantity is particularly small, it means that the deformation of PU elastomer is small when the external force is not too large; when the strain is between 0.2 and 0.6 , the slope of curve continues to increase with increasing strain, presenting the feature of up concave and showing hardening phenomenon. Based on the previous analysis, with the increasing compression amount, the stress and load has continued to increase. It means that the continuous improvement of strength, thereby improving the carrying capacity. At the end of the experiment, the test-piece can quick restore deformation, while plastic deformation does not occur. It has maintained rubber elasticity, so the polyurethane elastomer is polymeric material of high mechanical strength and excellent elastic property, as well as owns material nonlinearity.

\section{The constitutive model of the material}

What is first obtained is the relations between strain energy function and stress-strain. And then in the fitting process, the appropriate value for the initial model is selected. In order to reduce the 
variables, strain energy function in the conditions of uniform isotropic strain incompressible and unidirectional compression is simplified and get the elongation related expression on the basis of the engineering stress in strain energy function, and the relation curve between engineering stress and elongation curve is shown in Figure 2 to conduct fitting work, and select the most reasonable constitutive model, and then get the constitutive parameters. Finally the constitutive equations with good fitting results and less constitutive parameters are chosen as constitutive model of PU elastomer under uniaxial compression.

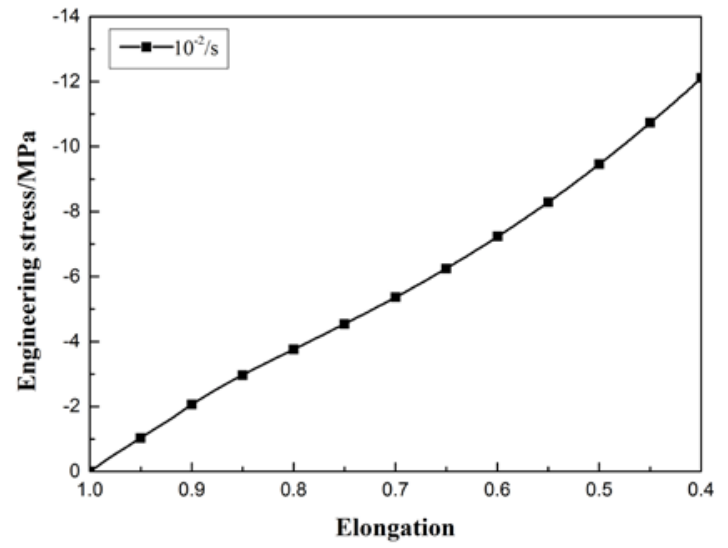

Fig. 2 The engineering stress and elongation curve of the specimen

In the quasi-static compression, and for the uniform isotropic incompressible rubber material, the relationship between first substantially invariant $\left(I_{1}\right)$ and second substantially invariant $\left(I_{2}\right)$ of the right Cauchy-Green deformation tensor and the main elongation $\lambda$ is that:

$$
\begin{aligned}
& I_{1}=\lambda^{2}+2 \lambda^{-1} \\
& I_{2}=2 \lambda+\lambda^{-2} \\
& \lambda=1+\varepsilon_{e}
\end{aligned}
$$

For the compression test, $\varepsilon_{e}$ is negative.

Here are some constitutive models [10-14], taking the formula (2) (3) into the models to achieve simplification, and the constitutive equation is obtained based on the model, and then the fitting curve is in Fig. 3. The constitutive parameters are obtained by the fitting results so that an appropriate constitutive model is selected.

Neo-Hookea model:

$$
\begin{aligned}
& W(\lambda)=\frac{1}{2} \mu\left(\lambda^{2}+2 \lambda^{-1}-3\right) \\
& \sigma_{e}=\frac{d W}{d \lambda}=A \lambda\left(1-\frac{1}{\lambda^{3}}\right)
\end{aligned}
$$

Mooney-Rivilin model:

$$
\begin{aligned}
& W(\lambda)=\mu_{1}\left(\lambda^{2}+\frac{2}{\lambda}-3\right)+\mu_{2}\left(2 \lambda+\frac{1}{\lambda^{2}}-3\right) \\
& \sigma_{e}=2\left(1-\frac{1}{\lambda^{3}}\right)\left(A_{1} \lambda+A_{2}\right)
\end{aligned}
$$

Yeoh strain-energy function:

$$
\begin{aligned}
& W(\lambda)=\mu_{1}\left(\lambda^{2}+\frac{2}{\lambda}-3\right)+\mu_{2}\left(\lambda^{2}+\frac{2}{\lambda}-3\right)^{2}+\mu_{3}\left(\lambda^{2}+\frac{2}{\lambda}-3\right)^{3} \\
& \sigma_{e}=2\left(\lambda-\frac{1}{\lambda^{2}}\right)\left[A_{1}+2 A_{2}\left(\lambda^{2}+\frac{2}{\lambda}-3\right)+3 A_{3}\left(\lambda^{2}+\frac{2}{\lambda}-3\right)^{2}\right]
\end{aligned}
$$

Trinomial of Mooney-Rivilin model: 


$$
\begin{aligned}
& W(\lambda)=\mu_{1}\left(\lambda^{2}+\frac{2}{\lambda}-3\right)+\mu_{2}\left(2 \lambda+\frac{1}{\lambda^{2}}-3\right)+\mu_{3}\left(\lambda^{2}+\frac{2}{\lambda}-3\right)\left(2 \lambda+\frac{1}{\lambda^{2}}-3\right) \\
& \sigma_{e}=2\left(1-\frac{1}{\lambda^{3}}\right)\left(A_{1} \lambda+A_{2}\right)+6 A_{3}\left(1-\frac{1}{\lambda^{3}}\right)\left(\lambda^{2}-\lambda+\frac{1}{\lambda}-1\right)
\end{aligned}
$$

Third-order deformation tenser invariant form:

$$
\begin{aligned}
& W(\lambda)=\mu_{1}\left(\lambda^{2}+\frac{2}{\lambda}-3\right)+\mu_{2}\left(2 \lambda+\frac{1}{\lambda^{2}}-3\right) \\
& +\mu_{3}\left(\lambda^{2}+\frac{2}{\lambda}-3\right)\left(2 \lambda+\frac{1}{\lambda^{2}}-3\right)+\mu_{4}\left(\lambda^{2}+\frac{2}{\lambda}-3\right)^{2} \\
& \sigma_{e}=2\left(1-\frac{1}{\lambda^{3}}\right)\left(A_{1} \lambda+A_{2}\right)+6 A_{3}\left(1-\frac{1}{\lambda^{3}}\right)\left(\lambda^{2}-\lambda+\frac{1}{\lambda}-1\right) \\
& +4 A_{4}\left(1-\frac{1}{\lambda^{3}}\right)\left(\lambda^{3}-3 \lambda+2\right)
\end{aligned}
$$

James-Green-Simpson form:

$$
\begin{aligned}
& W(\lambda)=\mu_{1}\left(\lambda^{2}+\frac{2}{\lambda}-3\right)+\mu_{2}\left(2 \lambda+\frac{1}{\lambda^{2}}-3\right)+\mu_{3}\left(\lambda^{2}+\frac{2}{\lambda}-3\right)\left(2 \lambda+\frac{1}{\lambda^{2}}-3\right) \\
& +\mu_{4}\left(\lambda^{2}+\frac{2}{\lambda}-3\right)^{2}+\mu_{5}\left(\lambda^{2}+\frac{2}{\lambda}-3\right)^{3} \\
& \sigma_{e}=2\left(1-\frac{1}{\lambda^{3}}\right)\left(A_{1} \lambda+A_{2}\right)+6 A_{3}\left(1-\frac{1}{\lambda^{3}}\right)\left(\lambda^{2}-\lambda+\frac{1}{\lambda}-1\right) \\
& +4 A_{4}\left(1-\frac{1}{\lambda^{3}}\right)\left(\lambda^{3}-3 \lambda+2\right)+2\left(1-\frac{1}{\lambda^{3}}\right)\left(\lambda^{3}-3 \lambda+2\right)\left[2 A_{4}+3 A_{5}\left(\lambda^{2}+\frac{2}{\lambda}-3\right)\right]
\end{aligned}
$$

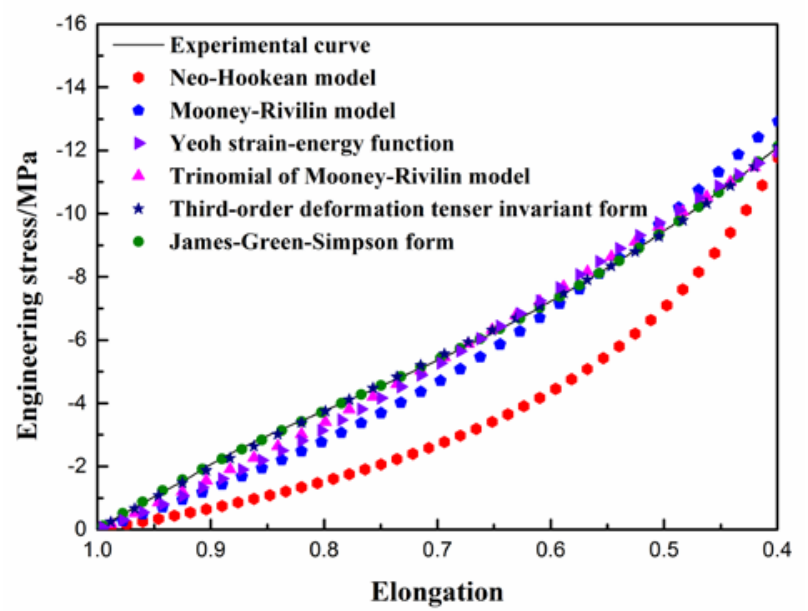

Fig .3 The fitting curve of the constitutive equation with experiment

In Figure 3, what can be apparently found is that the fitting result of Neo-Hookean model is not ideal, which can explain that the model does not apply to the fitting of the mechanical behaviors of large deformation compression. The fitting result of Mooney-Rivilin model, better than the one of the Neo-Hookean model, is still not ideal enough, in which the curve fitting deviates from the experimental curve greatly, so the Mooney-Rivilin model neither can be used to fit the mechanical behaviors of the large deformation compression. The fitting result of Yeoh strain energy function, better than Mooney-Rivilin model, which is slightly worse than Mooney-Rivilin in terms of trinomial, so fitting results in a small strain range is not ideal. Compared with the first few models, Trinomial of Mooney-Rivlin model can better fit the curve of the test, but the compressive strain between 0 and 0.3 , slightly deviates from the fitting curve. As a result, the trinomial of Mooney-Rivilin model faces minor errors in fitting results of large deformation compression so that 
it cannot achieve the desired effect. The fitting effect of third-order deformation tensor invariant form is very good and the fitting curve and the experimental curve essentially coincide. In addition, with four constitutive parameters, selecting third-order deformation tensor invariant form as PU elastomer in constitutive model of large deformation compression is a highly desirable result. The fitting effect of James-Green-Simpson form is very good, and even better than the fitting result of third-order deformation tensor invariant form. Therefore, James-Green-Simpson form is the best in fitting effect for now with the highest order and most parameters. Thus, we can really see that the fitting results of higher-order strain energy function would be better, then the third-order deformation tensor invariant form and James-Green-Simpson form can be the ideal constitutive model for PU elastomer, but James-Green-Simpson form, with five parameters, faces more computational and fitting difficulties. As a result, the third-order deformation tensor invariant form is a best option to describe the constitutive behavior of the PU elastomer.

The constitutive parameters obtained by fitting third-order deformation tensor invariant form: $A_{1}=8.8044, A_{2}=-5.3848, A_{3}=-0.0129, A_{4}=1.3457$.

The constitutive parameters and formula (4) is substituted into the model equation (14) so that the constitutive equation of PU elastomer compression in large deformation based on third-order deformation tensor invariants form:

$$
\sigma_{e}=5.38\left(1+\varepsilon_{e}\right)^{3}+1.38 \varepsilon_{e}-3.85-\frac{3}{2\left(1+\varepsilon_{e}\right)^{2}}
$$

\section{Summary}

After the study on the mechanical properties of PU elastomer under compression in large deformation, it is found in the experiments that PU elastomer exhibits hardening, and is always in the elastic stage, thus PU elastomer is with the properties like high strength, high carrying capacity, material nonlinearity and so on. Then in order to accurately describe the mechanical behavior of the materials, based on the strain energy function theory, constitutive model of compression mechanical properties of PU elastomer is obtained by fitting, and the constitutive equation of the PU elastomer under quasi-static compression is achieved on the basis of the selected third-order deformation tensor invariant form.

\section{References}

[1]. Shanxi Chemical Research Institute. Polyurethane Elastomer Manual. Beijing: Chemical Industry Press, 2001, p. 189-217. (in Chinese)

[2]. Boyce M C, Arruda E M. Constitutive Models of Rubber Elasticity: A Review. Rubber Chem Technol. Vol. 73 (2000) No. 3, p. 504-523.

[3]. Xiaofang Li, Xiaoxiang Yang. A review of elastic constitutive model for rubber materials. China Elastomerics. Vol. 15 (2005) No. 1, p. 50-58. (in Chinese)

[4]. Jianghua Tan, Wenbo Luo. Advances in Studies on Molecular Chain-network Based Constitutive Models of Rubber Materials. Materials Review. Vol. 22 (2008) No. 7, p. 31-34. (in Chinese)

[5]. Arruda E M, Boyce M C. A three-dimensional constitutive model for the large stretch behavior of rubber elastic materials. Journal of the Mechanics and Physics of Solids. Vol. 41 (1993) No. 2, p. 389-412.

[6]. Bo Song, Weinong Chen, Ming Cheng. Novel Model for Uniaxial Strain-Rate-Dependent Stress-Strain Behavior of Ethylene-Propylene-Diene Monomer Rubber in Compression or Tension. Journal of Applied Polymer Science. Vol. 92 (2004) No. 3, p. 1553-1558.

[7]. Hua Zhao, Minjie Wang, Lei Zhang, et al. Viscoelasticity modeling of polyurethane elastomer. Journal of Dalian University of Technology. Vol. 49 (2009) No. 4, p. 512-517. (in Chinese)

[8]. Kaisong Wu, Daping Xu, Yongfa Yan, et al. Data Processing Method of Rubber Testing Based on Incompressible Large deformation analysis. China Rubber Industry. Vol. 60 (2013) No. 7, p. 
400-403. (in Chinese)

[9]. GB/T7757-2009, Rubber, vulcanized or thermoplastic-Determination of compression stress-strain properties. Beijing: Standards Press of China, 2009. (in Chinese)

[10]. Mooney M J. A theory of large elastic deformation. Journal of Applied Physics. Vol. 11 (1940) No. 9, p. 582-592.

[11]. Treloar L R G. The elasticity of a network of long chain molecules III. Trans Faraday Soc. Vol. 42 (1946) No. 4, p. 83-94.

[12]. Tschoegl N W. Constitutive equations for elastomers. Journal of Polymer Science. Vol. 9 (1971) No. 7, p. 1959-1970.

[13]. Morman Jr K N, Pan T Y. Application of finite-element analysis in the design of automotive elastomeric components. Rubber Chemistry and Technology. Vol. 61 (1988) No. 3, p. 503-533.

[14]. Yeoh O H. Some Forms of the Strain Energy Function for Rubber. Rubber Chemistry and Technology. Vol. 66 (1993) No. 5, p. 754-771. 